\title{
Rendimiento académico y factores de salud ambiental asociados en estudiantes de una institución educativa pública de la región Cajamarca, Perú
}

\author{
Teófanez Adolfo Díaz-Ginéz ${ }^{\text {aa }}$, Julio César Sevilla-Excevio ${ }^{\text {1b }}$, Heber Silva-Díaz ${ }^{3 c}$
}

\section{RESUMEN}

Introducción. El examen PISA 2012 colocó al Perú en el último lugar, de un total de 65 países, en comprensión lectora y matemáticas. Objetivo. Determinar el rendimiento académico y los factores biológicos, sociales y ambientales asociados en estudiantes de la Institución Educativa Pública 16637 “Javier Heraud Pérez" del Distrito de Huabal, Provincia Jaén, Región Cajamarca, durante el 2018. Material y Métodos. Se realizó un estudio observacional analítico y prospectivo. La población estuvo constituida por 140 estudiantes de educación primaria y secundaria. La muestra fue censal. El rendimiento académico se obtuvo del registro de notas, la recolección factores biológicos incluyó análisis de laboratorio (parasitosis intestinal y anemia) y medidas antropométricas (nutrición) y para los factores sociales y ambientales a través de una encuesta a padres de familia y estudiantes. Resultados. El rendimiento académico fue destacado en el 1,4\%, logrado en el $89,3 \%$, en proceso en el $6,4 \%$ y en inicio el $2,9 \%$. El análisis bivariado demostró que el parasitismo intestinal fue el único factor asociado al bajo rendimiento académico $(p=0,016, R P=5,13$ e IC95=1,46 - 18,11). Asimismo se observó bajos niveles de anemia $(1,4 \%)$, peso saludable $(89,3 \%)$, grado de instrucción medio de los padres (primaria + secundaria 95,7\%), la asistencia regular al colegio $(99,3)$, y correcta disposición de los residuos sólidos en casa $(89,3 \%)$. Conclusiones. La mayoría de estudiantes obtuvieron un rendimiento logrado, no obstante, se observó al parasitismo intestinal como factor asociado en los estudiantes con bajo rendimiento académico, lo que evidencia la necesidad de atender la educación básica desde un enfoque integral.

Palabras clave: Rendimiento académico, Causalidad, parasitosis intestinales, Salud Ambiental (Fuente: DeCS-Bireme).

\footnotetext{
${ }^{1}$ Universidad Nacional Pedro Ruiz Gallo, Lambayeque, Perú..

${ }^{2}$ Laboratorio de Parasitología, Metaxénicas y Zoonosis, Hospital Regional Lambayeque, Lambayeque, Perú.

a Tecnólogo Médico, maestro en ciencias.

${ }^{\mathrm{b}}$ Pedagogo, maestro en ciencias.

${ }^{\mathrm{C}}$ Biólogo, doctor en ciencias.
}

Correspondencia: Teófanez Adolfo Díaz-Ginéz 


\section{Academic performance and associated environmental health factors in students of a public educational institution in the Cajamarca Region, Peru}

\section{ABSTRACT}

Introduction. The PISA 2012 exam placed Peru in the last place, out of a total of 65 countries, in reading comprehension and mathematics. Objective. To determine the academic performance and the associated biological, social and environmental factors in students of the Public Educational Institution 16637 "Javier Heraud Pérez" of the District of Huabal, Jaén Province, Cajamarca Region, during 2018. Material and methods. A cross-sectional and prospective study was carried out. The population was constituted by 140 students of primary and secondary education. The sample was census. The academic performance was obtained from the record of grades, the collection of biological factors included laboratory analysis (intestinal parasitosis and anemia) and anthropometric measures (nutrition); and for social and environmental factors through a survey of parents and students. Results. The academic performance was outstanding in $1.4 \%$, achieved in $89.3 \%$, in process in $6.4 \%$ and in beginning $2.9 \%$. The bivariate analysis showed that intestinal parasitism was the only factor associated with poor academic performance $(p=0.016, P R=5.13$ and IC95 $=1.46-18.11)$. Likewise, low levels of anemia $(1.4 \%)$, healthy weight $(89.3 \%)$, average level of education of the parents (primary + secondary 95.7\%), regular school attendance (99.3) were observed. , and correct disposal of solid waste at home (89.3\%). Conclusions. The majority of students obtained a successful performance. However, intestinal parasitism was observed as an associated factor in students with low academic performance, which evidences the need to attend basic education from an integral approach

Keywords: Academic performance, risk factors, intestinal diseases parasitic, environmental health (NLM-MESH).

\section{INTRODUCCIÓN}

El Informe de la Organización para la Cooperación y Desarrollo Económico (OCDE) en 2016, precisó sobre el Programa para la Evaluación Internacional de Alumnos (PISA) 2012, que; el Perú quedó en último lugar en las materias propósito de evaluación (matemática, lectura y ciencias), de un total de 65 países $^{(1)}$. Sobre esto, en el 2013 el Ministerio de Educación del Perú (MINEDU), comunicó que sólo el en comprensión lectora; y que el $24,0 \%$ y $51,4 \%$ lo alcanzan en instituciones estatales y no estatales respectivamente (2).

La salud ambiental refiere a las condiciones, influencias o fuerzas que rodean al hombre con relación al fomento, establecimiento y mantenimiento de la salud. Considerando que el humano es un ser complejo e integrado en sus aspectos biológicos, sociales, psicológicos y ambientales, estos impactan en la salud y el rendimiento académico de los estudiantes ${ }^{(3,4)}$.

Al respecto, las enfermedades parasitarias en niños de edad escolar en varias regiones del país continúan siendo un problema de salud pública importante ${ }^{(5,6)}$, por cuanto influyen directa y negativamente al estado nutricional antropométricos (7).

Un estudio en Ayacucho reportó que $46,2 \%$ y $38,6 \%$ de escolares procedentes de una zona rural y urbana, respectivamente, presentaron parasitismo por especies patógenas ${ }^{\left({ }^{8}\right)}$. Así también, estudios comunitarios en Chiclayo observaron frecuencias de $43,6 \%, 4,5 \%, 3,8 \%$ y $3,0 \%$ de infección por los protozoos patógenos Giardia lamblia, Entamoeba histolytica, Cryptosporidium spp. y Cyclospora cayetanensis, respectivamente ${ }^{(0,10)}$.
En cuanto al factor social, se ha observado que el nivel sociocultural de la población del país (medido como tasa de alfabetizados), está asociado con el mayor rendimiento académico de los alumnos ${ }^{(11)}$.

Del mismo modo, estudios alrededor del mundo (12,13), así como en Perú $(14,15)$, han demostrado el rol relevante del entorno social sobre el rendimiento académico.

A saber, las principales variables sociales reportadas son: la estructura y economía familiar, valoración familiar al estudio, ocupación familiar, así como aspectos culturales e institucionales.

En países en vía de desarrollo como Perú, el saneamiento ambiental y el acceso a los servicios básicos es aún problema vigente que afecta múltiples condiciones de la vida humana.

Al respecto, un estudio en el 2015 precisó que los determinantes sociales y ambientales que podrían afectar la primera infancia son el agua, higiene y saneamiento, vivienda y hacinamiento, educación de la madre, entre otros determinantes ${ }^{\left({ }^{16}\right)}$.

Los factores ambientales asociados a infección parasitaria intestinal por protozoos patógenos, y por lo tanto influyen en el rendimiento académico en niños de edad escolar, fueron: contacto con perros, vivir en casas con piso de tierra, hacer uso de letritas para eliminar sus excretas y el consumo de agua insalubre $(0,10)$.

No obstante el problema que representan los factores biológicos, sociales y ambientales en el rendimiento académico, ac tualmente se desconocen los factores biosocioambientales y la 
asociación de éstos al rendimiento académico en escolares de la provincia de Jaén, departamento de Cajamarca, Perú.

Razón por el cual, el presente estudio tuvo como objetivo determinar el rendimiento académico y los factores biosocioambientales asociados en estudiantes de la Institución Educativa Pública 16637 "Javier Heraud Pérez" del Distrito de Huabal, Provincia Jaén, Región Cajamarca, durante el 2018.

\section{MATERIAL Y MÉTODOS}

Diseño de la investigación. Diseño observacional (descriptivo), transversal analítico, y de temporalidad prospectiva.

Población y muestra. La población fue constituida por 140 estudiantes de educación primaria y secundaria de todos los grados de la Institución Educativa Pública 16637 "Javier Heraud Pérez" del distrito de Huabal, provincia Jaén, región Cajamarca. La muestra fue censal, incluyendo a todos los estudiantes (primer al sexto grado de primaria y primer al quinto grado de secundaria).

\section{Técnicas e instrumentos de recolección de datos}

Rendimiento académico. EL rendimiento académico se obtuvo del registro de notas que consta en el colegio, los mismos que fueron definidos como rendimiento académico destacado, logrado, en proceso e inicio, según su calificación de AD, A, B y $C$ respectivamente.

Factores Biológicos. Se consideró a la anemia, la parasitosis intestinal y el estado nutricional.

- La anemia demostrada mediante el dosaje de hemoglobina en cada estudiante. La lectura e interpretación se realizó en función de la guía de práctica clínica para diagnóstico y tratamiento de anemia del Ministerio de Salud (17).

- La parasitosis intestinal fue demostrada mediante el examen microscópico directo de muestras seriadas de heces (protozoos y helmintos) y el test de Graham (detección de oxiuros). La toma de muestra y ejecución de las técnicas siguieron las recomendaciones del Manual de procedimientos de laboratorio para el diagnóstico de las parasitosis del hombre ${ }^{(18)}$.

- Por otro lado, el estado nutricional se estudió mediante el Índice de Masa Corporal (IMC). El IMC se calculó siguiendo las recomendaciones del Centro para el Control y Prevención de las Enfermedades (CDC), para el cual se requirió la talla $(\mathrm{m})$ y peso $(\mathrm{Kg})$ de cada estudiante, además de su edad y sexo. Los IMC fueron registrados en las tablas de crecimiento de los CDC por edad (para niños o niñas), para obtener la categoría del percentil ${ }^{(12,13)}$. Los estudiantes se categorizaron como "bajo peso", "peso saludable", "sobrepeso-Obeso", cuando estuvieron con menos del percentil 5 , Percentil 5 hasta por debajo del percentil del percentil 5, Percentil 5 hasta por debajo del percentil 85 e igual o mayor al percentil 85 respectivamente.

Factores sociales y ambientales. En el factor social se evaluaron las siguientes variables: grado de instrucción de padres, asistencia regular al colegio y conocimiento de los padres sobre enfermedades parasitarias, parásitos perjudiciales y prevención de la parasitosis intestinales; y para el factor ambiental: tipo de agua de consumo, hábito de caminar descalzo y lavado de alimentos, eliminación de residuos sólidos, eliminación de roedores, eliminación de excretas y limpieza de la casa.

La recolección de datos de las variables de ambos factores se obtuvo a través de la aplicación de una encuesta estructurada a los padres de familia y estudiantes.

\section{Consideraciones éticas}

La participación de los estudiantes en el presente estudio fue realizada con la firma de un asentimiento informado por los mismos y un consentimiento informado por los padres. Asimismo, la aplicación de la encuesta a los padres también fue consentida.

También se realizó coordinaciones con el director de la mencionada institución para solicitar la autorización y relación de los alumnos. El estudio respetó los principios éticos fundamentales para la investigación descrita en la declaración de Helsinki. En todo momento se mantuvo la confidencialidad de la información, su acceso fue restringido a los investigadores y se usó solo con fines de investigación.

\section{Análisis de datos}

Se realizaron estadísticas descriptivas para la obtención de frecuencias absolutas y relativas de las variables cualitativas; y estimación de la mediana y rango intercuartílico de las variables cuantitativas, debido que todas presentaron distribución no paramétrica según la prueba de normalidad de Shapiro-Wilk. Asimismo, se realizaron pruebas inferenciales para el análisis bivariado entre el rendimiento académico y los factores biológicos, sociales y ambientales.

Se calculó el valor de "p" mediante la prueba exacta de Fisher o Chi cuadrado, según si la variable independiente fue dicotómica o categórica respectivamente. Para efectos del análisis bivariado de asociación con los factores esta variable fue re-categorizada a dos: rendimiento académico (RA) destacado-logrado y RA en proceso-inicio.

Asimismo, se calculó la Razón de Prevalencia y sus respectivos intervalos de confianza al 95\%; y se realizó un análisis multivariado de regresión logística. Se consideró significativo un valor de "p" menor de 0,05. El análisis estadístico requirió del uso de los softwares InfoStat y SPSS 21.0. 


\section{RESULTADOS}

Se evaluaron 140 estudiantes, desde primer grado de primaria hasta el quinto de secundaria.La muestra se caracterizó por presentar una mediana de edad de 12 años (rango intercuartílico [RIC] 9 a 14) y una frecuencia similar de varones $(50,7)$ y mujeres $(49,3)$; así como por presentar medianas de peso, talla, hematocrito y hemoglobina de $36 \mathrm{Kg}$ (RIC:2550); $1,41 \mathrm{~m}$ (RIC:1,126-1,53), 40,0 \% (RIC:38-44) y 12,9 g/dL (RIC:12,2-13,9), respectivamente.

Por otro lado el rendimiento académico de los estudiantes fue de aprendizaje destacado en el $1,4 \%(2 / 140)$, logrado en el $89,3 \%(125 / 140)$, en proceso en el $6,4 \%(9 / 140)$ y en inicio el 2,9\% (4/140).

En cuanto al factor biológico, la frecuencia de las variables y la asociación con el rendimiento académico (RA) se presentan en la tabla 01.

Cabe resaltar que el RA proceso-inicio estuvo asociado a la mayor frecuencia de parasitismo intestinal $(p=0,016, R P=5,13$ e IC95=1,46 - 18,11)
La parasitosis intestinal fue positivo en el 42,9\% (60/140) de los estudiantes examinados.

Los parásitos más frecuentes observados fueron Giardia lamblia $(15,7 \%)$ y Enterobius vermicularis (19,3\%). Ver tabla 02.

En cuanto al factor social, la variable grado de instrucción de los padres, predominó el nivel secundario $(52,1 \%)$, seguido por el primario $(43,6 \%)$.

Asimismo, se observó que el 99,3\% de estudiantes presentaron asistencia regular al colegio.

Sin embargo, el análisis bivariado no demostró asociación de estas variables con el rendimiento académico (Tabla 03).

Por otro lado, los factores ambientales se caracterizaron por un alto porcentaje de estudiantes que nos disponían de agua potable en sus viviendas (52,5\%), pero no se observó asociación con el rendimiento académico de los estudiantes (Tabla 04).

Tabla 1. Frecuencia de los factores biológicos y su asociación con el rendimiento académico en estudiantes de la Institución Educativa Pública 16637 “Javier Heraud Pérez” del distrito de Huabal, Provincia Jaén, región Cajamarca, 2018.

\section{Variable \\ RA proceso-inicio / Total \\ (\%)}

\section{Grupos etario (años)}

11 a menos

12 a 17

$$
74(52,9)
$$

Femenino

Masculino

Anemia

Sí

No

$$
\begin{gathered}
2(1,4) \\
138(98,6)
\end{gathered}
$$

\section{Parasitismo intestinal}

Positivo

Negativo

$$
\begin{aligned}
& 60(42,9) \\
& 80(57,1)
\end{aligned}
$$

\section{Nivel nutricional}

Bajo peso

Peso saludable

$$
1(0,7)
$$

Sobrepeso
$6 / 66(9,1)$

$7 / 74(9,5)$

$7 / 69(10,1)$

$6 / 71(8,5)$

$1 / 2(50,0)$

$12 / 138(8,7)$

$10 / 60(16,7)$

$3 / 80(3,8)$

$0 / 1(0,0)$

$10 / 125(8,0)$

$3 / 14(21,4)$
$14(10,0)$
Valor $\mathrm{p}^{*}$

$>0,999^{*}$
$0,778^{*}$

$0,178^{*}$

$0,016^{*}$

$0,316^{* *}$

(RA) Rendimiento académico, $\left({ }^{*}\right)$ Prueba exacta de Fisher, $\left({ }^{* *}\right)$ Prueba de Chi cuadrado 
Tabla 2. Tipo de parásitos intestinales en los estudiantes de la Institución Educativa Pública 16637 "Javier Heraud Pérez" del distrito de Huabal, Provincia Jaén, región Cajamarca, 2018.

\begin{tabular}{lcc}
\hline \multicolumn{1}{c}{ Parásitos intestinales } & N & $(\%)$ \\
\hline Protozoos & & \\
Giardia lamblia & 22 & $(15,7)$ \\
Entamoeba coli & 35 & $(25,0)$ \\
Endolimax nana & 4 & $(2,9)$ \\
Blastocystis hominis & 11 & $(7,9)$ \\
Retortamonas intestinalis & 2 & $(1,4)$ \\
Helmintos & & \\
Enterobius vermicularis & 27 & $(19,3)$ \\
\hline \hline
\end{tabular}

Tabla 3. Frecuencia de los factores sociales y su asociación con el rendimiento académico en estudiantes de la Institución Educativa Pública 16637 "Javier Heraud Pérez" del distrito de Huabal, Provincia Jaén, región Cajamarca, 2018.

\begin{tabular}{|c|c|c|c|}
\hline Variable & N (\%) & $\begin{array}{c}\text { RA en proceso-inicio / } \\
\text { Total (\%) }\end{array}$ & Valor $p$ \\
\hline \multicolumn{4}{|c|}{$\begin{array}{l}\text { Grado de instrucción de los } \\
\text { padres }\end{array}$} \\
\hline No tiene & $4(2,9)$ & $0 / 4(0,0)$ & \multirow{4}{*}{$0,753^{* *}$} \\
\hline Primaria & $61(43,6)$ & $6 / 61(9,8)$ & \\
\hline Secundaria & $73(52,1)$ & $7 / 73(9,6)$ & \\
\hline Superior & $2(1,4)$ & $0 / 2(0,0)$ & \\
\hline \multicolumn{4}{|c|}{ Asistencia regular al colegio } \\
\hline Sí & $139(99,3)$ & $13 / 139(9,4)$ & \multirow{2}{*}{$>0,999^{*}$} \\
\hline No & $1(0,7)$ & $0 / 1(0,0)$ & \\
\hline \multicolumn{4}{|c|}{$\begin{array}{l}\text { Conocimiento enfermedades } \\
\text { parasitarias }\end{array}$} \\
\hline Conoce & $98(70,5)$ & $8 / 98(8,2)$ & \multirow{2}{*}{$0,526^{*}$} \\
\hline Desconoce & $41(29,5)$ & $5 / 41(12,2)$ & \\
\hline \multicolumn{4}{|c|}{$\begin{array}{l}\text { Conocimiento de los parásit- } \\
\text { os perjudiciales }\end{array}$} \\
\hline Ameba & $18(12,9)$ & $2 / 18(11,1)$ & \multirow{4}{*}{$0,101^{* *}$} \\
\hline Ascaris & $39(27,9)$ & $1 / 39(2,6)$ & \\
\hline Giardia & $25(17,8)$ & $1 / 25(4,0)$ & \\
\hline Oxiuro & $58(41,4)$ & $9 / 58(15,5)$ & \\
\hline \multicolumn{4}{|c|}{$\begin{array}{l}\text { Conocimiento sobre pre- } \\
\text { vención de las parasitosis }\end{array}$} \\
\hline Control médico & $2(1,4)$ & $0 / 2(0,0)$ & \multirow{3}{*}{$0,025^{* *}$} \\
\hline Normas alimentación & $31(22,2)$ & $0 / 31(0,0)$ & \\
\hline Normas higiene & $107(76,4)$ & $13 / 107(12,2)$ & \\
\hline
\end{tabular}

(RA) Rendimiento académico $\left({ }^{*}\right)$ Prueba exacta de Fisher, $\left({ }^{* *}\right)$ Prueba de Chi cuadrado 
Tabla 4. Frecuencia de los factores ambientales y su asociación con el rendimiento académico en estudiantes de la Institución Educativa Pública 16637 “Javier Heraud Pérez” del distrito de Huabal, Provincia Jaén, región Cajamarca, 2018.

\section{\begin{tabular}{c} 
Variable \\
\hline Tipo de agua de consumo
\end{tabular}}

No potable

Potable

Hábitos de caminar descalzo

No

Sí

Correcta disposición de los residuos sólidos en casa

No

Sí

Hábito de lavado de alimen-

tos

No

Sí

Eliminación de los roedores

No

Sí

Correcta eliminación de las excretas

No

Sí

Enseñanza de lavado de manos al menor

Antes consumir alimentos

Después de baño

\section{Control parasitológico}

No

Sí

Frecuencia de limpieza de casa

Diario

Inter-diario

Quincenal

Semanal
$15(10,7)$

$125(89,3)$

$3(2,1)$

$137(97,9)$

$73(52,5)$

$66(47,5)$

$107(76,4)$

$33(23,6)$

$6(4,3)$

$134(95,7)$

$9(6,4)$

$131(93,6)$

$124(88,6)$

$16(11,4)$

$104(74,8)$

$35(25,2)$

$120(85,7)$

$16(11,4)$

$1(0,8)$

$3(2,1)$
$6 / 73(8,2)$

$7 / 66(10,6)$

9/107 $(8,4)$

$4 / 33(12,1)$

$0,733^{*}$

$0 / 15(0,0)$

$13 / 125(10,4)$

$0,361^{*}$

$0 / 3(0,0)$

$13 / 137(9,5)$

$>0,999^{*}$

$1 / 6(16,7)$

$12 / 134(9,0)$

$>0,999^{*}$

$1 / 9(11,1)$

$12 / 131(9,2)$

$>0,999^{*}$

10/124 $(8,1)$

$3 / 16(18,8)$

$0,171^{*}$

$11 / 104(10,6)$

$1 / 35(2,9)$

$0,191^{*}$

$10 / 120(8,3)$

$2 / 16(12,5)$

$0 / 1(0,0)$

$0,608^{* *}$

(RA) Rendimiento, $\left({ }^{*}\right)$ Prueba exacta de Fisher 


\section{DISCUSIÓN}

Casi $90 \%$ de los estudiantes demostraron un nivel de rendimiento académico Logrado. Estos resultados contrastan con características biosocioambientales favorables, como los muy bajos niveles de anemia, peso saludable, grado de instrucción de los padres, la asistencia regular al colegio, así como adecuadas prácticas higiénicas y de saneamiento ambiental.

Al respecto, en Puno, región del Perú que ha demostrado tener estudiantes de alto rendimiento académico, un estudio demostró que los siete hábitos de los niños con alto rendimiento fueron la lectura en casa, el acompañamiento familiar y la alimentación sana, la educación preescolar, hábito de estudio, hábito de hablar dos idiomas $\mathrm{y}$, el descanso y sueño ${ }^{200}$.

El factor biológico, y único, que se asoció al bajo rendimiento académico (en proceso-inicio) en las población estudiada fue la parasitosis intestinal; factor que estuvo presente el $42,9 \%$ de ellos.

Estos resultados concuerdan con los hallazgos encontrados por un estudio previo donde también verificaron que todos los estudiantes con bajo rendimiento académico tuvieron parásitos intestinales ${ }^{(21)}$.

Es conocido la influencia de las enfermedades parasitarias en la nutrición antropométrica de los niños (principalmente $G$. lamblia y helmintos), y por lo tanto influyen en el quehacer cognitivo (7).

Es importante notar que este grupo de enfermedades parasitarias afectan principalmente a la población de menor edad, así como se observó en el presente estudio, donde los estudiantes menores de 11 años tuvieron mayor probabilidad de presentar la enfermedad en comparación a los de mayor edad.

Este hallazgo ha sido observado previamente y descrito en parasitismo por Giardiasis en niños ${ }^{(9)}$. De hecho las enfermedades parasitarias continúan siendo un problema de salud pública vigente en nuestro país (i); donde los más afectados son los niños en edad escolar, quienes presentan altos niveles de parasitismo (8-10).

En este estudio, ninguna de las variables sociales investigadas demostró tener relación con el bajo rendimiento académico en la población estudiada.

El resultado podría deberse, por un lado, al bajo porcentaje de estudiantes en nivel de "proceso" o en "inicio", que impidió encontrar significancias estadísticas; y por otro, al contexto social adecuado que presentaron los estudiantes para mostrar el rendimiento académico observado.

Sin embargo, es necesario investigar otras variables sociales que podrían explicar en forma individual o múltiple el bajo rendimiento.No obstante la relación negativa de los factores sociales al rendimiento académico observado en este estudio, muchos estudios previos han revelado lo importantes que son para el desempeño académico ${ }^{(12,22,23)}$.

En Perú, se han identificado a estos factores como riesgo para el bajo desempeño académico (estado nutricional en delgadez, estructura familiar, ocupación de los padres) (15,24).

Si bien en este estudio ninguna de las variables ambientales estudiadas resultó relacionada al bajo rendimiento académico, el consumo de agua no potable estuvo asociado a la mayor frecuencia de parasitismo intestinal, y por lo tanto afectar indirectamente el rendimiento académico.

De hecho, los determinantes sociales y ambientales más importantes que podrían afectar la primera infancia son el agua, higiene, saneamiento, vivienda y hacinamiento ${ }^{(16)}$.

Al respecto, estudios previos han mostrado que varias circunstancias como el contacto continuo con perros, casa con piso de tierra, uso de letrinas para eliminación excretas, además del agua insalubre, pueden favorecer el parasitismo intestinal en niños de edad escolar $(6,10)$.

En este sentido, es importante incluir competencias a los profesionales de la salud, sobre las interrelaciones de los aspectos biológicos, psicológicos, sociales y ambientales, debido a que muestran cercana relación el rendimiento académico con la salud ${ }^{(3,4)}$.

Por otra parte, el resultado negativo de predicción del rendimiento académico debido a factores biosocioambientales mediante el análisis multivariado, demuestra la necesidad de estudiar otras variables explicativas en la población estudiada.

El presente estudio tuvo algunas limitaciones. Los datos referidos al rendimiento escolar fueron obtenidos de una fuente secundaria (registro de notas), razón por el cual es posible el sesgo de sobreestimación del buen rendimiento escolar (nivel logrado), debido a subjetividad o forma de evaluación del docente responsable.

Asimismo, la cantidad relativamente reducida de estudiantes estudiados, no permitió establecer un mayor número o con mayor evidencia relaciones de causalidad entre los factores estudiados y el rendimiento académico.

Por razones logísticas y de factibilidad no se describió el factor psicológico y otras variables sociales y ambientales importantes de los estudiantes propósito de estudio.

Estudios previos han manifestado la importancia de los aspectos psicológicos como autoestima, violencia y la motivación que surge de la valoración familiar al estudio, para el buen rendimiento académico ${ }^{(3,12)}$. 
En conclusión, la mayoría de estudiantes obtuvieron un rendimiento logrado, No obstante, se observó al parasitismo intestinal como factor asociado en los estudiantes con bajo rendimiento académico, lo que evidencia la necesidad de atender la educación básica desde un enfoque integral.

Conflicto de interés. Los autores declaran no tener conflicto de interés.

Fuentes de financiamiento. Autofinanciado.

\section{REFERENCIAS BIBLIOGRÁFICAS}

1. Organización para la Cooperación y el Desarrollo Económicos. PISA Estudiantes de bajo rendimiento: por qué se quedan atrás y cómo ayudarles a tener éxito . 2016 [cited 2018 Feb 26]. 46 p. Available from: http://www.oecd.org/pisa/keyfindings/PISA-2012-Estudiantes-de-bajo-rendimiento.pdf

2. Ministerio de Educación. Resultados ECE 2013 | UMC Oficina de Medición de la Calidad de los Aprendizajes. 2013 [cited 2018 Mar 15]. Available from: http://umc.minedu.gob.pe/resultados-ece-2013-3/

3. Castro Bosch M, Espinosa Rodríguez R, Victoria Pujals N, Durán García F. Principios morfofuncionales biológicos y sociambientales en la salud. Educ médica Super. 2016;30(1)

4. Castro Bosch M, Espinosa Rodríguez R, Pujals Victoria N, Durán García F, Díaz Cruz L. Lo social en el currículo de formación de profesionales de la salud. Rev Cuba Educ médica Super. 2012;26(3):450-8.

5. Ipanaque-Chozo J, Seclen-Bernabe E, Bustamante-Canelo O, Aguilar-Gamboa F-R, Mera-Villasis K, Vergara-Espinoza M, et al. Enteropatógenos predominantes en diarreas agudas y variables asociadas en niños atendidos en el Hospital Regional Lambayeque, Perú. Rev Horiz Med. 2017;17(1):38-44.

6. Silva-Díaz H, Iglesias-Osores SA, Failoc-Rojas VE. Enteroparasitosis: Un problema vigente de salud pública en el norte del Perú. Rev Cuerpo Med HNAAA. 2017;10(3):173-4.

7. Solano Li, Acuña I, Barón MA, Moróm de Salim A, Sánchez A. Influencia de las parasitosis intestinales y otros antecedentes infecciosos sobre el estado nutricional antropométrico de niños en situación de pobreza. Parasitol Latinoam. 2008;63:12-9.

8. Espejo Ramos RP. Parasitosis intestinal en estudiantes del nivel primario de Huancayo al 2014. Apunt Cienc Soc. 2014;04(01):78-86.

9. Silva-Díaz H, Monteza-Salazar J, Rentería-Valle CA. Elisa y Examen Microscópico Directo en la Detencción de Giardia en Muestras Fecales de Niños en Chongoyape, Chiclayo, Perú. Rev Exp en Med del Hosp Reg Lambayeque. 2015;1(1):6-10.

10. Vilches-Berríos GN, Rentería-Valle CA, Monteza-Salazar JL, Silva-Díaz $\mathrm{H}$. Coccidiosis y amibiasis intestinal en niños de edad escolar de un distrito de Lambayeque, Perú. Rev Medica Hered. 2018;29(1):5-10.

11. Castañeda E, Fabara E, Murillo O, Cueto S, Hernandez ML, Román M, et al. Investigación iberoamericana sobre eficacia escolar. Bogotá; 2007 [cited 2018 Feb 24]. 376 p. Available from: https:// www.uam.es/personal_pdi/stmaria/jmurillo/documentos/IIEE.pdf

12. Barca Lozano A, Porto Rioboo A, Brenlla Blanco JC, Morán Fraga $\mathrm{H}$, Barca Enríquez E. Contextos familiares y rendimiento escolar en el alumnado de educación secundaria. Int J Dev Educ Psychol. 2007;(2):197-218.

13. González-Pineda JA. El rendimiento escolar. Un análisis de las variables que lo condicionan. Rev Galego-Portuguesa Psicoloxía e Educ. 2003;7(8):247-58

14. Lip C, Rocabado F. Determinantes sociales de la salud en Perú. MINSA, editor. Lima; 2005 [cited 2018 Mar 18]. 87 p. Available from: http://www.minsa.gob.pe/dgsp/archivo/salud_mental_documentos/12_determinantes_sociales_salud.pdf

15. Muelle $L$. Factores de riesgo en el bajo desempeño académico y desigualdad social en el Perú según PISA 2012. Apunt Rev Ciencias Soc. 2016;43(79):9-45.
16. Raineri F, Confalone Gregorian M, Barbieri ME, Zamorano MS, Gorodisch R, Ortiz Z. Determinantes sociales y ambientales para el desarrollo de los niños y niñas desde el período del embarazo hasta los 5 años. 2015 [cited 2018 Feb 24]. 82 p. Available from: https://www. unicef.org/argentina/spanish/SALUD_PBPrimeralnfancia_web(1). pdf

17. Ministerio de salud. Guia de práctica clínica para el diagnóstico y tratamiento de la anemia por deficiencia de hierro en niñas, niños y adolescentes en establecimientos de salud del primer nivel de atención. Lima; 2016 [cited 2018 Feb 24]. 26 p. Available from: http://bvs. minsa.gob.pe/local/MINSA/3932.pdf.

18. Beltrán Fabían de Estrada $M$, Tello Casanova R, Náquira Velarde $C$ Manual de procedimientos de laboratorio para el diagnóstico de los parásitos intestinales del hombre. Instituto Nacional del Perú, editor. Lima; 2003 [cited 2018 Jan 3]. 90 p. Available from: http://bvs.minsa. gob.pe/local/ins/165_nt37.pdf.

19. Central for Desease Control and Prevention. Acerca del índice de masa corporal para niños y adolescentes | Peso Saludable | DNPAO | CDC. [cited 2018 Mar 8]. Available from: https://www.cdc.gov/ healthyweight/spanish/assessing/bmi/childrens_bmi/acerca_indice_ masa_corporal_ninos_adolescentes.html

20. Vilca Apaza HM, Mamani Apaza WW. Los siete hábitos de los niños con alto rendimiento académico en Puno: análisis desde el contexto y tipo de gestión. Comuni@cción. 2017;8(1):48-60.

21. Santana Parrales A. Parasitosis y rendimiento académico de los alumnos del cuarto "A" y "B" de la unidad educativa Grad. Miguel Iturralde de Portoviejo, 2009. Tesis Universidad Tecnológica Equinocial; 2010: 196p

22. Cervini R. Desigualdades socioculturales en el aprendizaje de Matemática y Lengua de la Educación Secundaria en Argentina: un modelo de tres niveles. Reli - Rev Electrónica Investig y Evaluación Educ. 2002;8(2):135-58.

23. Cervini R. Desigualdades en el logro académico y reproducción cultural en Argentina. Un modelo de tres niveles. Rev Mex Investig Educ. 2002;7(16):445-500.

24. Arzapalo-Salvador F, Pantoja-Villalobos K, Romero-López J, Farro-Peña G. Estado nutricional y rendimiento escolar de los niños de 6 a 9 años del Asentamiento Humano Villa Rica - Carabayllo Lima Perú 2011. Rev enferm Hered. 2011;4(1):20-6.

Revisión de pares: Recibido: 06/02/2019 Aceptado: 28/03/2019 